\title{
Patients' subjective quality of life during involuntary treatment in psychiatric hospitals Joanna Rymaszewska
}

\author{
Address: Department of Psychiatry, Wroclaw Medical University, Pasteura 10, 50-367 Wroclaw, Poland \\ from WPA Thematic Conference. Coercive Treatment in Psychiatry: A Comprehensive Review \\ Dresden, Germany. 6-8 June 2007 \\ Published: 19 December 2007 \\ BMC Psychiatry 2007, 7(Suppl I):S33 doi:10.1 I86/I47|-244X-7-SI-S33
}

This abstract is available from: http://www.biomedcentral.com//47I-244X/7/SI/S33

(c) 2007 Rymaszewska; licensee BioMed Central Ltd.

\section{Background}

To test the hypotheses that (1) quality of life of psychiatric patients admitted legally involuntarily and legally voluntarily but feeling coerced to admission differs; (2) higher level of aggression, and lower level of social functioning are associated with lower quality of life (QoL); (3) there are several predictors for the improvement of QoL within the 3-month assessment period.

\section{Methods}

Each patient from 7 European centers ( $\mathrm{n}=978,48 \%$ females) was assessed at 3 time-points: within the first seven days, 4 and 12 weeks after admission to psychiatric hospitals. The patient's subjective quality of life was assessed with the Short Assessment of Quality of Life (MANSA). The 12 subjective items assessing different life domains were used in the analyses. Several further factors were analyzed by regression models: psychopathology, perceived coercion, social functioning, and satisfaction with treatment.

\section{Results}

Quality of Life increased significantly with time. Higher baseline QoL was associated with being employed, and better educated as well as with lower BPRS scores and social disability. After one month and at the follow-up the higher QoL was strongly associated with education, employment, psychopathology and the period since the first psychiatric hospitalization. Diagnosis and center did not influence QoL. QoL was lower in the involuntary group in comparison to the voluntary group at each measure point.

\section{Conclusion}

Other factors than center and diagnosis influence quality of life: social functioning, perceived coercion and severity of the disorder. 